\title{
Chaos in a Fractional-Order Single-Machine Infinite-Bus Power System and Its Adaptive Backstepping Control
}

\author{
Zhanhong Liang, Jinfeng Gao \\ School of Electrical Engineering, Zhengzhou University, Zhengzhou, China \\ Email: liangzhanhong@zzu.edu.cn
}

How to cite this paper: Liang, Z.H. and Gao, J.F. (2016) Chaos in a Fractional-Order Single-Machine Infinite-Bus Power System and Its Adaptive Backstepping Control. International Journal of Modern Nonlinear Theory and Application, 5, 122-131. http://dx.doi.org/10.4236/ijmnta.2016.53013

Received: August 18, 2016

Accepted: September 24, 2016

Published: September 27, 2016

Copyright (c) 2016 by authors and Scientific Research Publishing Inc. This work is licensed under the Creative Commons Attribution International License (CC BY 4.0). http://creativecommons.org/licenses/by/4.0/

\begin{abstract}
This paper has numerically studied the dynamical behaviors of a fractional-order single-machine infinite-bus (FOSMIB) power system. Periodic motions, perioddoubling bifurcations and chaotic attractors are observed in the FOSMIB power system. The existence of chaotic behavior is affirmed by the positive largest Lyapunov exponent (LLE). Based on the fractional-order backstepping method, an adaptive controller is proposed to suppress chaos in the FOSMIB power system. Numerical simulation results demonstrate the validity of the proposed controller.
\end{abstract}

\section{Keywords}

Power System, Fractional Calculus, Chaos, Backstepping Method

\section{Introduction}

As a mathematical branch with a history of over 300 years, fractional calculus and its applications to physics and engineering have attracted increasing attentions in recent years [1] [2]. Fractional calculus provides a good instrument to describe the memory, hereditary, non-locality and self-similarity properties of various materials and processes. Many chaotic systems, such as Lorenz system [3], Chua's system [4], Duffing system [5], Rössler system [6], Chen system [7] and so on, still remain chaotic when their equations become fractional.

Chaotic phenomena have been observed in power systems during the past few decades [8]-[13]. Chaos causes electromechanical oscillations to behave randomly, which are harmful to the secure and stable operation of power systems, and even produce undesired negative consequences, such as angle divergence, voltage collapse and system splitting [14]. So far, almost all the studies of dynamics of power systems are concerned 
with the integer-order models, and there are little research results on fractional modeling and control design of power systems. Tan et al. studied the dynamics of a fractional-order interconnected power system and found that the system became chaotic when the fractional order is no less than 0.88 [15]. Sun and Li investigated the chaotic and bifurcation phenomena in a fractional-order three-bus power system and the existence of chaos was demonstrated for different orders [16].

In this paper, we numerically investigate the chaotic dynamics of a fractional-order single-machine infinite-bus (FOSMIB) power system. Period-doubling bifurcation and chaos are observed in FOSMIB power system and the existence of chaos is confirmed by evaluating the largest Lyapunov exponent (LLE). Based on the fractional-order backstepping method, an adaptive controller is presented to suppress chaos in the FOSMIB power system, and the effectiveness of the proposed controller is proved by the numerical simulation results.

The rest of the paper is organized as follows. Some definitions and lemmas about fractional calculus are introduced in Section 2. The dynamics of the FOSMIB power system are analyzed in Section 3. An adaptive controller is designed using the fractional-order backstepping method to suppress chaos in the FOSMIB power system in Section 4. Finally, conclusions are addressed in Section 5.

\section{Preliminaries}

There are several different definitions of fractional derivatives. The most appropriate one for practical problems is the Caputo definition. The Caputo fractional derivative is given by

$$
D_{t}^{q} x(t)=\left\{\begin{array}{l}
\frac{1}{\Gamma(m-q)} \int_{0}^{t}(t-\tau)^{(m-q-1)} x^{(m)}(\tau) \mathrm{d} \tau, m-1<q<m, \\
\frac{\mathrm{d}^{m}}{\mathrm{~d} t^{m}} x(t), q=m,
\end{array}\right.
$$

where $m$ is integer and $\Gamma(\cdot)$ is the Gamma function.

The Caputo fractional derivative satisfies the following properties:

$$
\begin{aligned}
& D_{t}^{q} C=0, \\
& D_{t}^{q}\left(k_{1} x(t)+k_{2} y(t)\right)=k_{1} D_{t}^{q} x(t)+k_{2} D_{t}^{q} y(t),
\end{aligned}
$$

where $C, k_{1}$ and $k_{2}$ are real constants.

Lemma 1. [17]-[19] Consider the fractional-order system

$$
D_{t}^{q} x(t)=f(x(t)),
$$

where $q \in(0,1)$ and $x \in R^{n}$. The equilibrium point $x^{*}$ of system (3) is locally asymptotically stable if all the eigenvalues $\lambda$ of the Jacobian matrix $J=\partial f /\left.\partial x\right|_{x^{*}}$ satisfy

$$
|\arg (\lambda)|>\frac{q \pi}{2} .
$$

Lemma 2. [20] Let $x(t) \in \mathbb{R}$ be a continuous differentiable function. Then, at any 
instant the following inequality holds

$$
\frac{1}{2} D_{t}^{q} x^{2}(t) \leq x(t) D_{t}^{q} x(t), \forall q \in(0,1) .
$$

A continuous function $\alpha:[0, t) \rightarrow[0,+\infty)$ is referred as class- $K$ if it is strictly increasing and $\alpha(0)=0 \quad$ [21].

Lemma 3. (Fractional-order extension of Lyapunov direct method [22]) Let $x=0$ be an equilibrium point of the nonautonomous fractional-order system

$$
D_{t}^{q} x(t)=f(t, x(t))
$$

with initial condition $x(0)$. Assume that $V(t, x(t))$ is a Lyapunov candidate and $\alpha_{i}(i=1,2,3)$ are class-K functions. Then $x=0$ is asymptotically stable if the following conditions hold

$$
\begin{gathered}
\alpha_{1}(\|x\|) \leq V(t, x(t)) \leq \alpha_{2}(\|x\|), \\
D_{t}^{q} V(t, x(t)) \leq-\alpha_{3}(\|x\|),
\end{gathered}
$$

where $q \in(0,1)$ and $\|\cdot\|$ denotes an arbitrary norm.

\section{The FOSMIB Power System}

In [12] Chen et al. analyzed the angle dynamics of the classical single-machine infinite-bus (SMIB) power system, which is governed by the so-called swing equation

$$
M \ddot{\theta}+D \dot{\theta}+P_{\max } \sin \theta=P_{m},
$$

where $M$ is the moment of inertia, $D$ is the damping constant, $P_{\max }$ is the maximum power of generator and $P_{m}=A \sin \omega t$ is the power of the machine.

Let $x=\theta$ and $y=\dot{\theta}$, then Equation (9) can be rewritten as

$$
\left\{\begin{array}{l}
\dot{x}=y, \\
\dot{y}=-c y-\beta \sin x+f \sin \omega t,
\end{array}\right.
$$

where $c=D / M, \beta=P_{\max } / M$ and $f=A / M$ are positive constant parameters. When $c=0.5, \beta=1, \omega=1$ and $f=2.41$, the SMIB power system is chaotic.

Here, we consider the fractional-order single-machine infinite-bus (FOSMIB) power system

$$
\left\{\begin{array}{l}
D_{t}^{q} x=y, \\
D_{t}^{q} y=-c y-\beta \sin x+f \sin \omega t,
\end{array}\right.
$$

where $0<q \leq 1$ is the fractional order. When $q=1$, system (11) is the original integer-order SMIB power system.

The autonomous system (11) (as $f=0$ ) has two equilibrium points: $O(0,0)$ and $E(\pi, 0)$. For the equilibrium point $O$, the Jacobian matrix is

$$
J=\left[\begin{array}{cc}
0 & 1 \\
-\beta & -c
\end{array}\right],
$$

and its eigenvalues are 


$$
\lambda_{1,2}=\left\{\begin{array}{l}
\frac{-c \pm \sqrt{c^{2}-4 \beta}}{2}, \beta \leq \frac{c^{2}}{4}, \\
\frac{-c \pm i \sqrt{4 \beta-c^{2}}}{2}, \beta>\frac{c^{2}}{4} .
\end{array}\right.
$$

In both cases, $\left|\arg \left(\lambda_{1,2}\right)\right|>q \pi / 2$. According to Lemma $1, O$ is asymptotically stable. For the equilibrium point $E$, the Jacobian matrix is

$$
J=\left[\begin{array}{cc}
0 & 1 \\
\beta & -C
\end{array}\right],
$$

and its eigenvalues are

$$
\lambda_{1,2}=\frac{-c \pm \sqrt{c^{2}+4 \beta}}{2} .
$$

It can be seen that $\lambda_{1}>0$ and $\left|\arg \left(\lambda_{1}\right)\right|=0<q \pi / 2$. In accordance with Lemma $1, E$ is unstable.

\section{Dynamic Analysis of the FOSMIB Power System}

In this section, we use the Adams-Bashforth-Moulton predictor-corrector algorithm proposed by Diethelm et al. in [22]-[24] to solve the FOSMIB power system (11). The dynamics are numerically analyzed by means of bifurcation diagrams, phase portraits and Lyapunov exponents. In the following simulations, parameter $f$ is chosen as bifurcation parameter and the other parameters are fixed at $c=0.5, \beta=1, \omega=1$. The initial conditions are selected as $x(0)=1, y(0)=-0.3$.

First, let $q=0.95$, and vary $f$ from 2.4 to 3.5 . The corresponding bifurcation diagram is plotted in Figure 1(a), from which a period-doubling route to chaos can be found. To confirm chaos, the largest Lyapunov exponent (LLE) is calculated using Wolf

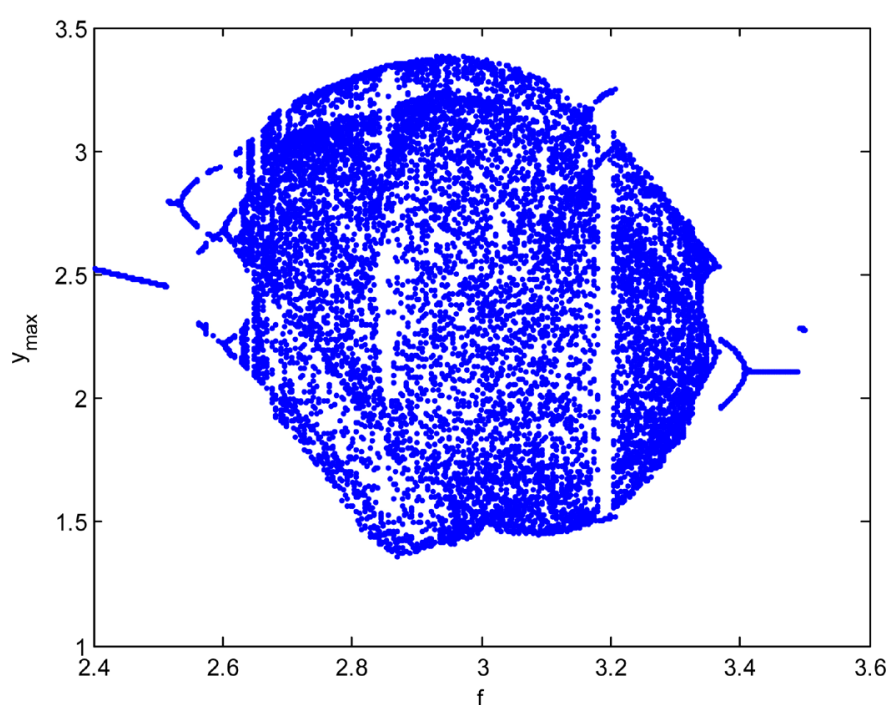

(a)

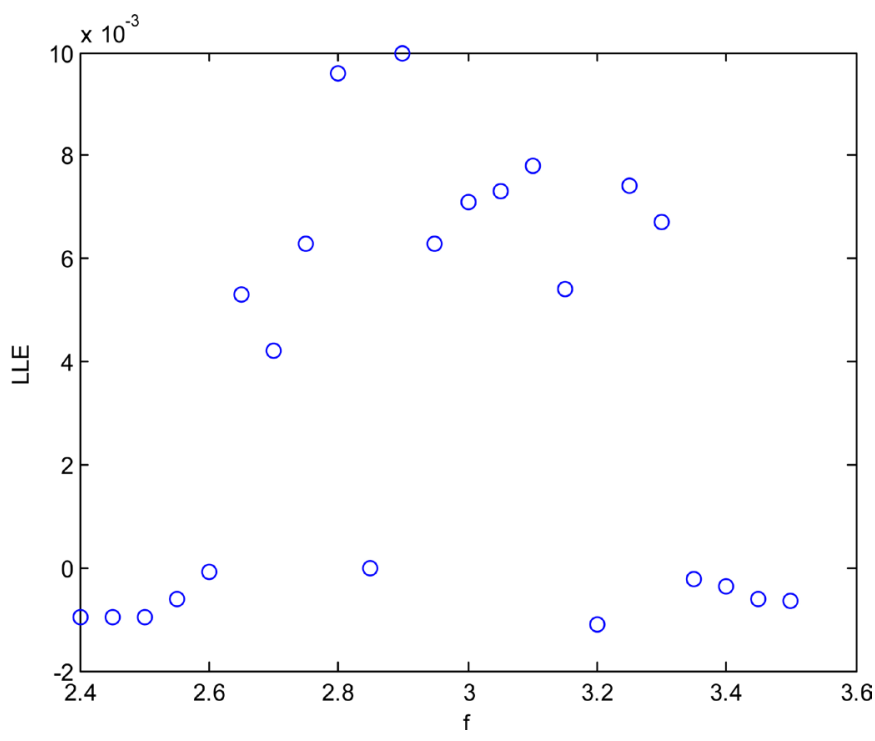

(b)

Figure 1. Bifurcation diagram and the LLE versus $f$ for $q=0.95$ : (a) Bifurcation diagram; (b) The LLE. 
algorithm [25] and plotted in Figure 1(b). The FOSMIB power system is chaotic over most of the range $f \in[2.65,3.3]$, where the LLEs are positive. The phase portraits for different values of $f$ are plotted in Figure 2. With the increase of $f$ from 2.4, period-1, period- 2 and period- 4 orbits are obtained at $f=2.5, f=2.55$ and $f=2.61$, respectively. After a cascade of period-doubling bifurcations, the system loses its stability and enters chaos at $f=2.65$. As $f$ increases further, the system becomes stable again via inverse period-doubling bifurcations.

Now, let $c=0.5, \quad \beta=1, \omega=1, f=2.8$ and vary $q$ from 0.87 to 1 . The resulting bifurcation diagram is plotted in Figure 3(a), which indicates period-doubling bifurcations and chaos. The fractional-order SMIB power system is chaotic over most of the range $q \in[0.92,1]$, where the LLEs are positive as shown in Figure $3(b)$. The phase portraits for different values of $q$ are plotted in Figure 4. With the increase of $q$ from 0.87, period-1, period- 2 and period- 4 orbits are obtained at $q=0.88, q=0.893$ and $q=0.913$, respectively. As $q$ increases further, after a cascade of period-doubling bifurcations, a chaotic attractor is obtained at $q=0.92$.

\section{Adaptive Backstepping Control of Chaos}

In this section, an active controller is designed using fractional-order backstepping method to suppress chaos in the FOSMIB power system and stabilize it to the unstable equilibrium point $E(\pi, 0)$.

\subsection{Controller Design}

Consider the controlled FOSMIB power system

$$
\left\{\begin{array}{l}
D_{t}^{q} x=y, \\
D_{t}^{q} y=-c y-\beta \sin x+f \sin \omega t+u,
\end{array}\right.
$$

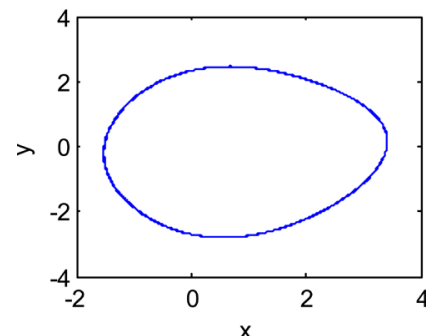

(a)

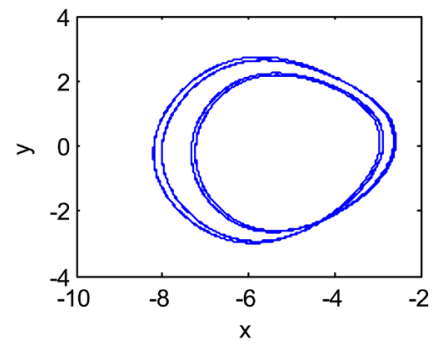

(c)

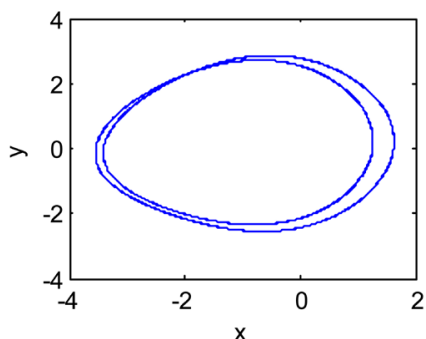

(b)

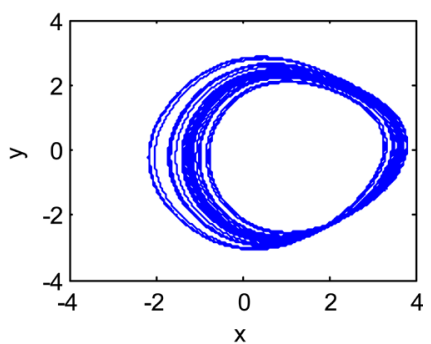

(d)

Figure 2. Phase portraits for different values of $f$. (a) $f=2.5$; (b) $f=2.55$; (c) $f=2.61$; (d) $f=2.65$. 


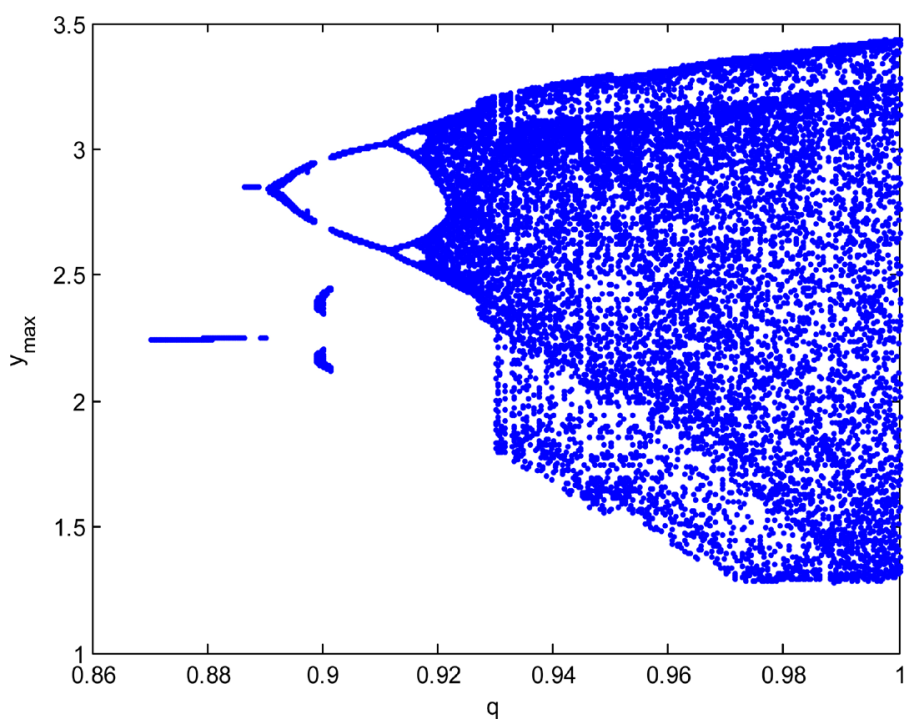

(a)

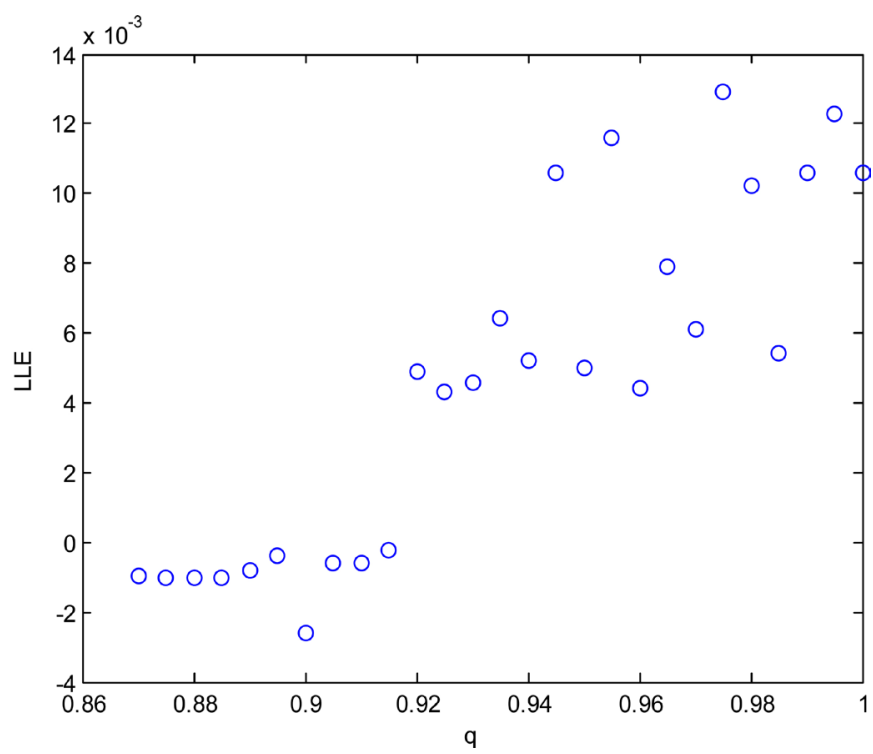

(b)

Figure 3. Bifurcation diagram and the LLE versus $q$ for $f=2.8$ : (a) Bifurcation diagram; (b) The LLE.

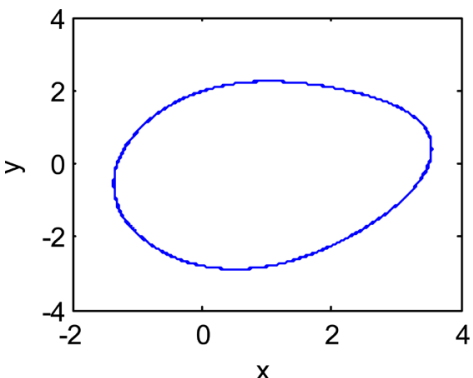

(a)

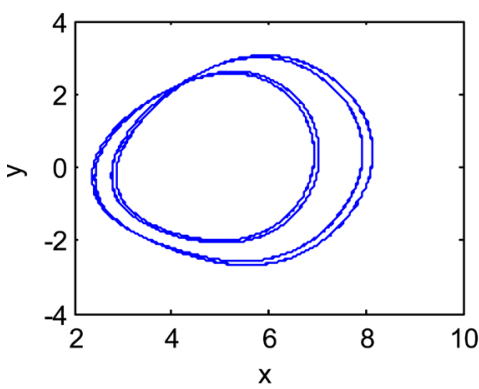

(c)

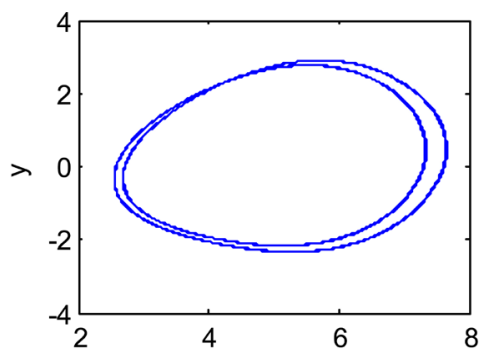

$\mathrm{X}$
(b)

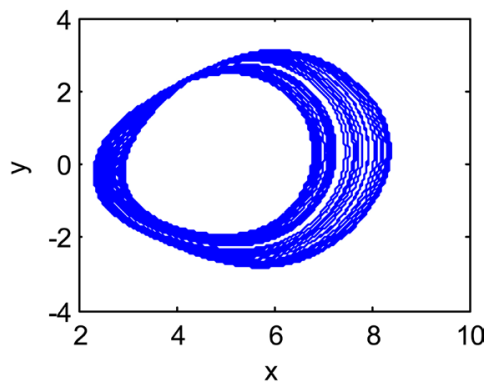

(d)

Figure 4. Phase portraits for different values of $q$ : (a) $q=0.88$; (b) $q=0.893$; (c) $q=0.913$; (d) $q=$ 0.92 .

where $q \in(0,1)$ and the parameter $f$ is unknown. The backstepping design procedure consists of two steps.

Step 1. Define $e_{1}=x-\pi$. Its derivative is given by

$$
D_{t}^{q} e_{1}=D_{t}^{q} x=e_{2}+\alpha_{1}
$$

where $e_{2}=y-\alpha_{1}, \alpha_{1}$ is the virtual control to be defined later.

Select the candidate Lyapunov function as 


$$
V_{1}=\frac{1}{2} e_{1}^{2},
$$

Now, applying Lemma 2, it can be found that

$$
D_{t}^{q} V_{1} \leq e_{1}\left(e_{2}+\alpha_{1}\right) \text {. }
$$

Define the virtual control $\alpha_{1}$ as

$$
\alpha_{1}=-c_{1} e_{1},
$$

where $c_{1}$ is a positive constant, which leads to $e_{2}=y+c_{1} e_{1}$. Substituting Equation (20) into Equation (17) and inequality (19), we have

$$
\begin{gathered}
D_{t}^{q} e_{1}=-c_{1} e_{1}+e_{2}, \\
D_{t}^{q} V_{1} \leq-c_{1} e_{1}^{2}+e_{1} e_{2} .
\end{gathered}
$$

Step 2. The derivative of $e_{2}$ is expressed as

$$
D_{t}^{q} e_{2}=D_{t}^{q} y+c_{1} D_{t}^{q} e_{1}=-c_{1}^{2} e_{1}+c_{1} e_{2}-c y-\beta \sin x+(f-\hat{f}) \sin \omega t+\hat{f} \sin \omega t+u,
$$

where $\hat{f}$ is the estimate of $f$. Choose the candidate Lyapunov function as

$$
V_{2}=V_{1}+\frac{1}{2} e_{2}^{2}+\frac{1}{2 k}(f-\hat{f})^{2},
$$

where $k$ is a positive constant, which can adjust the speed of the adaptive law. Using Lemma 2, it can be found that

$$
\begin{aligned}
D_{t}^{q} V_{2} \leq & D_{t}^{q} V_{1}+e_{2} D_{t}^{q} e_{2}-\frac{1}{k}(f-\hat{f}) D_{t}^{q} \hat{f} \\
\leq & -c_{1} e_{1}^{2}+e_{2}\left[\left(1-c_{1}^{2}\right) e_{1}+c_{1} e_{2}-c y-\beta \sin x+\hat{f} \sin \omega t+u\right] \\
& +(f-\hat{f})\left(e_{2} \sin \omega t-\frac{1}{k} D_{t}^{q} \hat{f}\right) .
\end{aligned}
$$

Choose the control input and the adaptive law as

$$
\begin{gathered}
u=-\left(1-c_{1}^{2}\right) e_{1}-\left(c_{1}+c_{2}\right) e_{2}+c y+\beta \sin x-\hat{f} \sin \omega t, \\
D_{t}^{q} \hat{f}=k e_{2} \sin \omega t,
\end{gathered}
$$

where $C_{2}$ is a positive constant. Substituting Equation (26) and Equation (27) into Equation (23) and inequality (25), we have

$$
\begin{gathered}
D_{t}^{q} e_{2}=-e_{1}-c_{2} e_{2}+(f-\hat{f}) \sin \omega t, \\
D_{t}^{q} V_{2} \leq-c_{1} e_{1}^{2}-c_{2}^{2} e_{2} .
\end{gathered}
$$

According to Lemma 3, the closed-loop error system is asymptotically stable at the origin $(0,0)$. It means that, with the proposed controller and adaptive law, the FOSMIB power system is asymptotically stable at the equilibrium point $E_{2}(\pi, 0)$.

\subsection{Simulation Results}

In the simulation, the fractional order $q$ is equal to 0.95 . The parameters of system (16) are taken as $c=0.5, \beta=1, \omega=1$ and $f=2.66$. The parameters of the controller 
(26) and the adaptive law (27) are chosen as $c_{1}=c_{2}=0.5$ and $k=2$. The initial conditions are taken as $x(0)=1, y(0)=-0.3$. The initial parameter estimate is given by $\hat{f}(0)=0.5$. The closed-loop system consisted of Equations ((16), (26) and (27)) is solved by using the predictor-corrector algorithm. The simulation results are shown in Figure 5 .

The time-domain waveforms the states of the controlled system (16) are shown in Figure 5(a) and Figure 5(b). The FOSMIB power system has experienced chaotic behavior before the controller is put into effect. By activating the controller $u$ at $t=20 \mathrm{~s}$, the chaotic behavior is suppressed and the controlled system converges to the equilibrium point $E_{2}(\pi, 0)$ quickly. The parameter estimate $\hat{f}$ is converged to $f$ as shown in Figure 5(c) and the controller $u$ is bounded as shown in Figure 5(d). From Figure 5, it can be seen that the proposed controller is feasible for suppressing chaos in the FOSMIB power system.

\section{Conclusion}

In this paper, we have numerically investigated the FOSMIB power system. The parameter $f$ and the fractional order $q$ are selected as bifurcation parameters respectively. Complex dynamical behaviors, such as periodic orbits, period-doubling bifurcations and chaotic attractors, are observed in the FOSMIB power system. The LLE is calculated using Wolf algorithm to confirm the existence of chaos. Furthermore, by exploiting the fractional-order backstepping method, we propose an adaptive controller to suppress chaos in the FOSMIB power system. The effectiveness of the presented controller is verified by numerical simulation results.

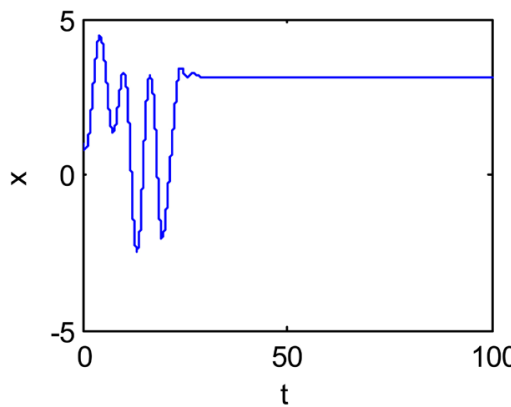

(a)

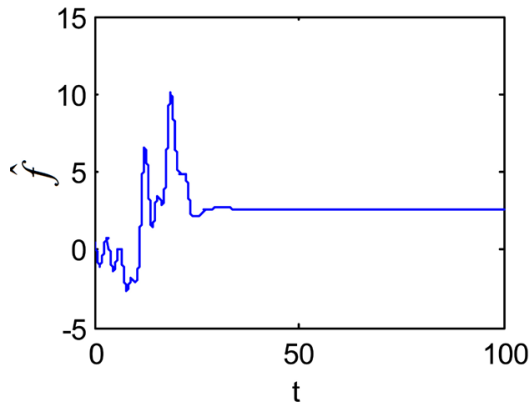

(c)

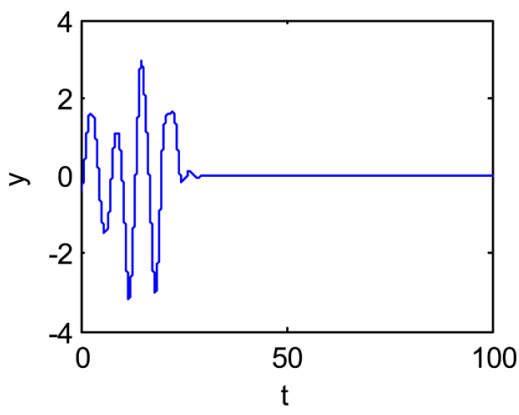

(b)

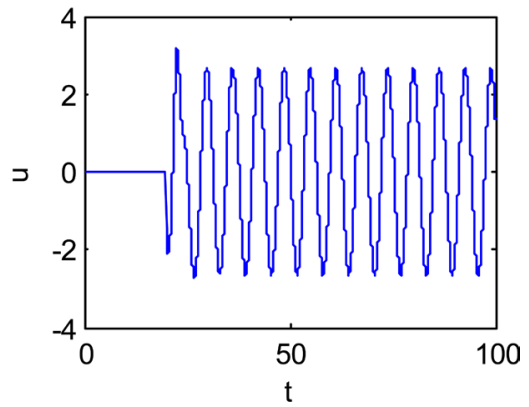

(d)

Figure 5. The time-domain waveforms of the controlled system (16). 


\section{Acknowledgements}

The work was supported by the Natural Science Foundation of Henan Province, China (Grant No. 14A120005) and Excellent Young Scientist Development Foundation of Zhengzhou University, China (Grant No. 1421319086).

\section{References}

[1] Podlubny, I. (1999) Fractional Differential Equations. Academic Press, New York.

[2] Hilfer, R. (2001) Applications of Fractional Calculus in Physics. World Scientific, New Jersey.

[3] Grigorenko, I. and Grigorenko, E. (2003) Chaotic Dynamics of the Fractional Lorenz System. Physical Review Letters, 91, Article ID: 034101. http://dx.doi.org/10.1103/PhysRevLett.91.034101

[4] Hartley, T.T., Lorenzo, C.F. and Qammer, H.K. (1995) Chaos in a Fractional Order Chua's System. IEEE Transactions on Circuits and Systems CAS-I, 42, 485-490. http://dx.doi.org/10.1109/81.404062

[5] Gao, X. and Yu, J.B. (2005) Chaos in the Fractional Order Periodically Forced Complex Duffing's Oscillators. Chaos, Solitons and Fractals, 24, 1097-1104. http://dx.doi.org/10.1016/j.chaos.2004.09.090

[6] Li, C.G. and Chen, G.R. (2004) Chaos and Hyperchaos in the Fractional-Order Rössler Equations. Physica A, 341, 55-61. http://dx.doi.org/10.1016/j.physa.2004.04.113

[7] Li, C.P. and Peng, G.J. (2005) Chaos in Chen's System with a Fractional Order. Chaos, Solitons and Fractals, 22, 443-450. http://dx.doi.org/10.1016/j.chaos.2004.02.013

[8] Kopell, N. and Washburn, R.B. (1982) Chaotic Motions in the Two-Degree-Of-Freedom Swing Equations. IEEE Transactions on Circuits and Systems, 29, 738-746. http://dx.doi.org/10.1109/TCS.1982.1085094

[9] Lee, B. and Ajjarapu, V. (1993) Period-Doubling Route to Chaos in an Electrical Power System. IEE Proceedings C-Generation, Transmission and Distribution, 140, 490-496. http://dx.doi.org/10.1049/ip-c.1993.0071

[10] Chiang, H.D., Conneen, T.P. and Flueck, A.J. (1994) Bifurcations and Chaos in Electric Power Systems: Numerical Studies. Journal of the Franklin Institute, 331, 1001-1036. http://dx.doi.org/10.1016/0016-0032(94)90095-7

[11] Ji, W. and Venkatasubramanian, V. (1996) Hard-Limit Induced Chaos in a Fundamental Power System Model. International Journal of Electrical Power and Energy Systems, 18, 279-295. http://dx.doi.org/10.1016/0142-0615(95)00066-6

[12] Chen, H.K., Lin, T.N. and Chen, J.H. (2005) Dynamic Analysis, Controlling Chaos and Chaotification of a SMIB Power System. Chaos, Solitons and Fractals, 22, 1307-1315. http://dx.doi.org/10.1016/j.chaos.2004.09.081

[13] Gholizadeh, H., Hassannia, A. and Azarfar, A. (2013) Chaos Detection and Control in a Typical Power System. Chinese Physics B, 22, 10503-10507. http://dx.doi.org/10.1088/1674-1056/22/1/010503

[14] Yu, Y.X., Jia, H.J., Li, P. and Su, J.F. (2003) Power System Instability and Chaos. Electric Power Systems Research, 65, 187-195. http://dx.doi.org/10.1016/S0378-7796(02)00229-8

[15] Tan, W., Zhang, M. and Li, Z.P. (2011) Chaotic Oscillation of Interconnected Power System and Its Synchronization. Journal of Hunan University of Science and Technology (Natural Science Edition), 26, 74-78. (In Chinese) 
[16] Sun, F.Y. and Li, Q. (2014) Dynamic Analysis and Chaos of the 4D Fractional-Order Power System. Abstract and Applied Analysis, 2014, Article ID: 534896.

http://dx.doi.org/10.1155/2014/534896

[17] Ahmed, E., El-Sayed, A.M.A. and El-Saka, H.A.A. (2007) Equilibrium Points, Stability and Numerical Solutions of Fractional-Order Predator-Prey and Rabies Models. Journal of Mathematical Analysis and Applications, 325, 542-553. http://dx.doi.org/10.1016/j.jmaa.2006.01.087

[18] Tavazoei, M.S. and Haeri, M. (2007) A Necessary Condition for Double Scroll Attractor Existence in Fractional-Order Systems. Physics Letters A, 367, 102-113. http://dx.doi.org/10.1016/j.physleta.2007.05.081

[19] Tavazoei, M.S. and Haeri, M. (2008) Chaotic Attractors in Incommensurate Fractional Order Systems. Physica D: Nonlinear Phenomena, 237, 2628-2637. http://dx.doi.org/10.1016/j.physd.2008.03.037

[20] Aguila-Camacho, N., Duarte-Mermoud, M.A. and Gallegos, J.A. (2014) Lyapunov Functions for Fractional Order Systems. Communications in Nonlinear Science and Numerical Simulation, 19, 2951-2957. http://dx.doi.org/10.1016/j.cnsns.2014.01.022

[21] Li, Y., Chen, Y.Q. and Podlubny, I. (2009) Mittag-Leffler Stability of Fractional Order Nonlinear Dynamic Systems. Automatica, 45, 1965-1969. http://dx.doi.org/10.1016/j.automatica.2009.04.003

[22] Diethelm, K., Ford, N.J. and Freed, A.D. (2002) A Predictor-Corrector Approach for the Numerical Solution of Fractional Differential Equations. Nonlinear Dynamics, 29, 3-22. http://dx.doi.org/10.1023/A:1016592219341

[23] Diethelm, K. (1997) An Algorithm for the Numerical Solution of Differential Equations of Fractional Order. Electronic Transactions on Numerical Analysis, 5, 1-6.

[24] Diethelm, K. and Ford, N.J. (2002) Analysis of Fractional Differential Equations. Journal of Mathematical Analysis and Applications, 265, 229-248. http://dx.doi.org/10.1006/jmaa.2000.7194

[25] Wolf, A., Swift, J.B., Swinneya, H.L. and Vastano, J.A. (1985) Determining Lyapunov Exponents from a Time Series. Physica D: Nonlinear Phenomena, 16, 285-317. http://dx.doi.org/10.1016/0167-2789(85)90011-9

\section{Submit or recommend next manuscript to SCIRP and we will provide best service for you:}

Accepting pre-submission inquiries through Email, Facebook, LinkedIn, Twitter, etc.

A wide selection of journals (inclusive of 9 subjects, more than 200 journals)

Providing 24-hour high-quality service

User-friendly online submission system

Fair and swift peer-review system

Efficient typesetting and proofreading procedure

Display of the result of downloads and visits, as well as the number of cited articles

Maximum dissemination of your research work

Submit your manuscript at: http://papersubmission.scirp.org/

Or contact ijmnta@scirp.org 